\title{
Heat storage materials based on polymers modified with functionalized carbon nanotubes
}

\author{
Alexander Shchegolkov ${ }^{1, *}$, Tatyana Dyachkova ${ }^{1}$, and Elena Burakova ${ }^{1}$ \\ ${ }^{1}$ Tambov State Technical University, 392000, Tambov, Russia
}

\begin{abstract}
The article presents studies of nanomodified materials based on paraffin waxes with polyethylene as heat-storage materials. Accumulation of thermal energy is possible when converting electrical energy into thermal energy. In this case, the accumulation of thermal energy can occur as a central zone of nanomodified materials, and at its ends. This is due to the location of the electrodes in nanomodified materials. When CNT was mixed with polyethylene and the mixture was subsequently heated on the surface of a CNT, a homogeneous PE layer of a special structure was formed. The presented feature of the interaction can be considered as a special case of functionalization.
\end{abstract}

\section{Introduction}

The development and creation of nanostructured composite materials is currently one of the most urgent areas of scientific and applied research. Areas of application of such materials present fairly stringent requirements, which correspond to polymer composites, which have been actively studied in recent years $[1,2]$. Due to the wide possibilities of modifying, it is possible to create polymer composite materials that are resistant both to mechanical influences [3] and erosion wear [4], and also, upon reaching the percolation threshold with the addition of carbon-containing nanomaterials, high electrical conductivity [5,6]. Carbon nanomaterials, in particular, are also very promising as additives for the effective elasticization of heat-resistant binders, since they allow simultaneously increasing the deformation characteristics and the modulus of elasticity of the polymer matrix [7]. The key problem in the preparation of nanocomposites is the uniform distribution of the nanomaterial in the polymer matrix in connection with their propensity to agglomerate [8]. One way to increase the affinity of carbon nanostructures to modifiable matrices is functionalization through the formation of various types of surface groups, which contributes to a significant increase in the efficiency of nanomaterial use and a reduction in its expenditure norms [9]. Recently, carbon nanotubes and their modifications act as nanomodifiers of various polymers, the performance characteristics of the resulting composites will depend on their properties and morphology. Thus, the introduction of their insignificant amount into the polymer matrix allows to significantly improve the physical and mechanical characteristics (bending strength, compression, thermal conductivity, electrical conductivity) of the polymer composites obtained, which is why carbon

\footnotetext{
*Corresponding author: Energynano@yandex.ru
} 
nanostructured materials (CNMs) are so attractive as fillers of composite materials. However, each field of application of nanomodifiers poses certain requirements to them (diameter, length, defectiveness, specific surface, etc.). Competent selection of nanomodifier (UNM), the ability to direct its synthesis with the required characteristics will achieve the best affinity of the nanomodifier with a polymer matrix (ABS plastic, polyethylene, fluoropolymer). Despite the fact that UNM learned how to produce on an industrial scale, the problem of implementing a directed synthesis of carbon nanostructures with the required characteristics remains to this day. In the process of synthesis of CNM by the CVD method, a powerful tool for controlling their parameters is the catalyst, since it is at its active centers that the nanostructure (nanofiber, nanotube) is nucleated and further grown. It is known that the diameter of the nanostructure formed depends on the size of the active sites of the catalyst, and the length of the catalyst system, the content of amorphous carbon and the specific yield of the nanoproduct, so it is important to be able to obtain an efficient selective catalyst for the synthesis of CNM. Experimental study of the features of the process of obtaining effective, selective catalysts for the synthesis of CNM by the thermal decomposition method will make it possible to carry out directed synthesis of a nanomodifier (with the required characteristics) for the polymers under consideration. The developed technique of introduction of the obtained nanomodifier into the polymer matrix will allow to obtain an innovative competitive composite with improved characteristics. The obtained knowledge and research results will make a significant contribution to the integration of scientific developments and real production in the field of nanocomposite creation. It is known that the traditional methods of activation of heterogeneous catalytic systems (heat treatment, oxidation or reduction at high temperatures in the environment of various gases) do not always provide the required properties of the catalyst due to the formation of active centers of various types, including those that catalyze undesirable side reactions in the process of synthesis. To avoid undesirable reactions during the synthesis of catalytic systems, other methods of activation are often resorted to (the action of microwave radiation, IR spectra, magnetic fields, etc.). The efficiency of thermal accumulators is associated with the use of heat-accumulating materials, which determine their main parameters. The most suitable material is paraffin [10]. The use of paraffin in its pure form is not very effective in view of its low thermal conductivity. At the same time, there are works [10-15] in indicating the possibility of an increase in thermal conductivity due to the introduction of materials having a high thermal conductivity, but this direction allows the production of passive thermal accumulators that are not controlled, and charge / discharge modes are associated with the operation of a source of thermal energy and consumer, which makes such a process uncoordinated. One of the most effective ways to improve the thermophysical parameters of paraffins is to modify them with nanomaterials that have high thermal conductivity. For a long time, the approaches to creating heat-storage materials, in which the application of materials possessing high thermal conductivity as additives for less heat-conducting materials, was laid, which is the classical rule of additivity. In this respect, the higher the content and thermal conductivity of the input material, the stronger the thermal properties of the matrix will change. Among the wide range of nanomaterials capable of increasing thermal conductivity, the following are distinguished: metal nanoparticles [10, 11], metal foams [12], carbon materials (fibers, nanotubes and graphenes) [14-17]. In these papers, the main attention is paid solely to the influence of nanomaterials or on the thermal conductivity or electrical conductivity, while the issues of heat release under the influence of electric current are not considered. The flow of electrical current in the composite is affected by electrical resistance, and the spread of thermal energy - thermal conductivity. The research tasks include research of heat release in a polymer composite based on paraffin and polyethylene modified with carbon nanotubes. 


\section{Methods and Materials}

New approaches to the activation of CNM synthesis catalysts will be used to obtain Ni$\mathrm{MgO}$ (composition 8: 2) and Co-Mo-Al2O3-MgO catalyst. At the stage of catalyst preparation, a short-term energy effect (ultrasound, electromagnetic field) is expected to be applied to the solution of the initial components (precatalyst), which contribute to increasing their efficiency. Preliminary studies of the effect of non-traditional activation methods of $\mathrm{NiMgO}(9: 1)$ synthesis catalyst of CNM synthesis (ultrasonic processing with a frequency of $22 \mathrm{kHz}$ and the action of a weak electromagnetic field of $0.12 \mathrm{~T}$ ) on its efficiency have shown that short-term energy supply to the pre-catalyst promotes an increase in the specific yield of UNM g / gkat) by 30\%, while the homogeneity of the synthesized nanomaterial is observed. As part of the process of functionalization, nitrated CNMs were obtained. As a modifiable polymer, a composite containing polyethylene and paraffin was used. Method for studying thermophysical characteristics To study the distribution of the temperature field on the surface of nanomodified material (NM), a contactless method for measuring temperature was used. As the instrument on the basis of which the presented method was implemented, the thermal imager Testo 875 was used, which has the following characteristics: - Detector size $160 \times 120$ pixels $\bullet$ Lens $32^{\circ}$ x $23^{\circ}$ • Built-in digital camera $\cdot$ Temperature range from -20 to $+350^{\circ} \mathrm{C}$ In this case, the degree of blackness of the ETH is preliminarily determined. The surface of the NM, in order to exclude the reflection of infrared rays in the sides not perpendicular to the imager of the thermal imager, as well as to prevent the imposition of infrared rays, was equalized by imposing an even and smooth surface, followed by heating of the NM and dense pressing. In addition to the measures presented to eliminate the effect of reflection of infrared rays from the investigated NM surface, the thermal imager was directed at different angles. Repeat measurements were at least five times. The data of the distribution of the temperature field were recorded in the memory of the thermal imager. Processing of the received data was carried out in the specialized program Testo IRSoft 2.7.

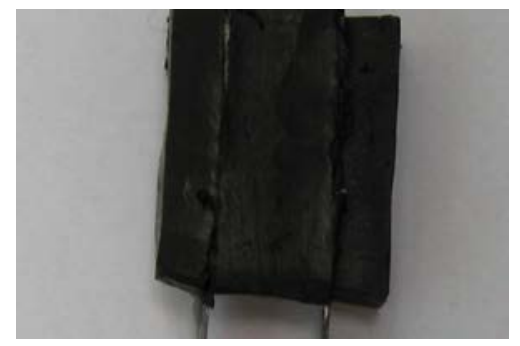

Fig. 1. General view of NM (plastic polymer)

To study the distribution of equipotential lines on the surface of the heating element HM, the Aktak 1097 multimeter was used. Using the probes of the multimeter, the distribution of the equipotential lines on the surface of the NM was determined on the surface. 


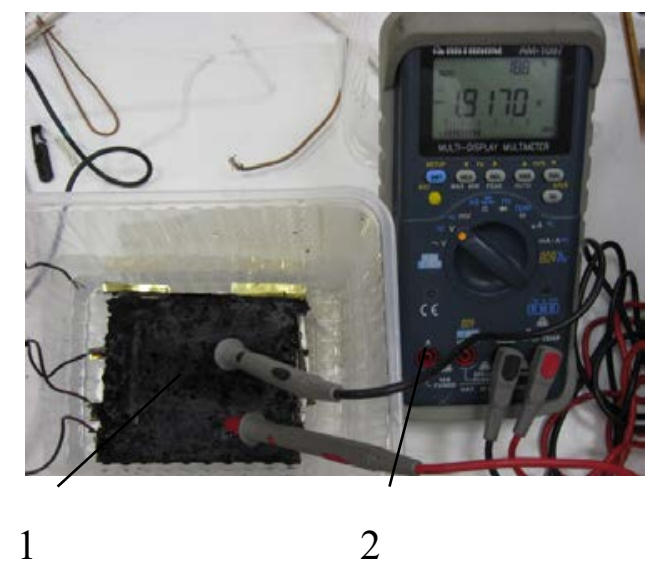

Fig. 2. Study of the electric field strength on the surface of NM (1 - NM; 2 - multimeter Aktak 1097)

\section{Results and discussion}

When mixing CNT with polyethylene and then heating the mixture on the surface of a CNT, a homogeneous PE layer of a special structure was formed. The presented feature of the interaction can be considered as a special case of functionalization. Figure 3 shows the distribution of the equipotential field on the NM surface with the end position of the supply electrodes.

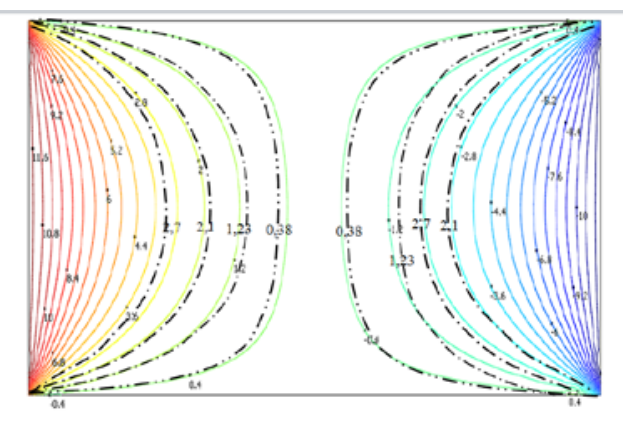

Fig. 3. Distribution of equipotential lines on the surface of NM

Figure 4 shows the distribution of the equipotential field on the surface of the NM with the lateral arrangement of the supply electrodes.

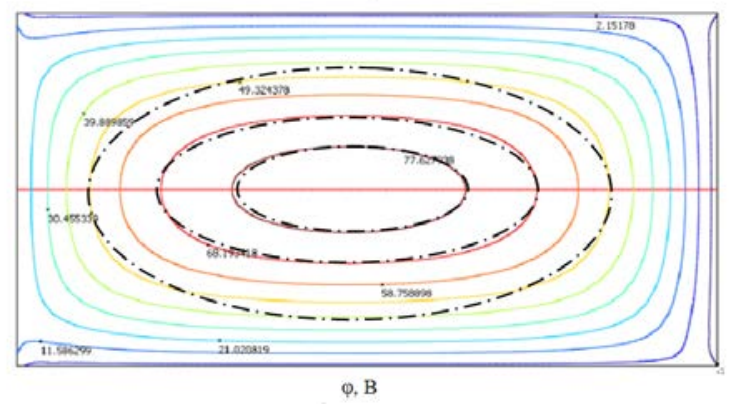

Fig. 4. Distribution of equipotential lines on the surface of the NM 
The three-dimensional distribution of the potential field on the surface of the NM is shown in Fig. 5.

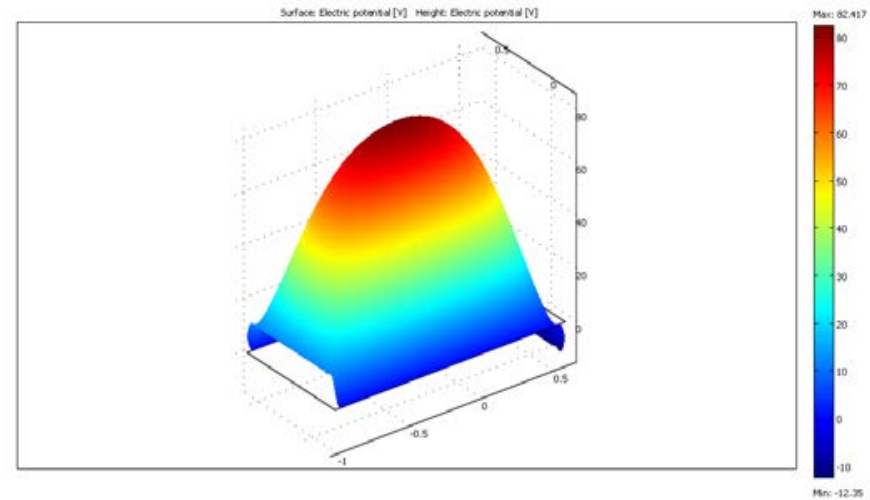

Fig. 5. 3d distribution of equipotential lines on the surface of NM

Figure 6 shows the distribution of the temperature field with a distance between the electrodes equal to $1 \mathrm{~cm}$. The temperature difference is $10^{\circ} \mathrm{C}$.

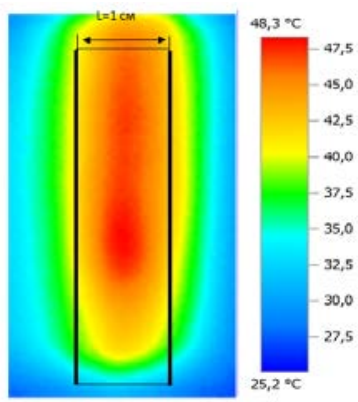

Fig. 6. Distribution of the temperature field on the surface of the NM

\section{Conclusions}

The conducted studies have shown that it is possible to use nanomodified materials as heatstorage materials. Accumulation of thermal energy is possible when converting electrical energy into thermal energy. In this case, the accumulation of thermal energy can occur as the central zone of the NM, and at its ends. This is due to the location of the electrodes. When CNT was mixed with polyethylene and the mixture was subsequently heated on the surface of a CNT, a homogeneous PE layer of a special structure was formed. The presented feature of the interaction can be considered as a special case of functionalization.

The reported research was funded by Russian Foundation for Basic Research and the government of the region of the Russian Federation, grant № 18-43-680012 p_a

\section{References}

1. Hessam Yazdani, Benjamin E. Smith, Kianoosh Hatami // Composites Part A: Applied Science and Manufacturing, (82) 2016 
2. Hamid Ghasemi, Roham Rafiee, Xiaoying Zhuang, Jacob Muthu, Timon Rabczuk // Journal of Magnetism and Magnetic Materials, (401) 2016.

3. J. Gao, Ch. Li, U. Shilpakar, Y. Shen // Materials and Design, (86) 2015

4. Jinhu Chen, Ian M. Hutchings, Tong Deng, Michael S.A. Bradley, Krzysztof K.K. Koziol // Carbon, (73) 2014

5. Mohammed H. Al-Saleh, Haya K. Al-Anid, Yazan A. Hussain // Composites: Part A., (46) 2013

6. Wolfgang Bauhofer, Josef Z. Kovacs // Composites Science and Technology, (69) 20097. E.N. Kablov, S.V. Kondrashov, G.Yu. Yurkov Carbon Nanotube Composites, (6415) 2006

9. Punetha V. D., Rana S., Yoo H. J., Chaurasia A et al. // Progress in Polymer Science. (67) 2017

10. Fan L., Khodadadi J.M. Renewable and sustainable energy reviews, (15) 2011

11. Warzoha R. J., Zhang D., Feng G., Fleischer A. S. CARBON, (61) 2013

12. Warzoha R. J., Fleischer A. S. Applied Energy, (154) 2015

13. Li M., Guo Q., Nutt S. Solar energy, (146) 2017

14. Zhang N., Yuan Ya., Yuan Ya., Cao X., Yang X. Solar Energy, (110) 2014.

15. Renteria J.D., Nika D.L., Balandin A.A. Applied sciences, (4) 2014

16. Kant K., Shukla A., Sharma A., Biwole P.H. Solar Energy, (146) 2017

17. Liu X., Rao Z. Thermochimica acta, (647) 2017 